\title{
Survey on Item Based and User Based Recommendation System in Cloud
}

\author{
Pinal Patel ${ }^{1}$, Pooja Jardosh ${ }^{2}$ \\ Silver oak collage of engineering and technology, Gujarat technological university, Ahmedabad, Gujarat, India
}

\begin{abstract}
Today there is a big variety of different approaches and algorithms of recommendation. In this paper we describe the recommendation system related research and then introduce various techniques and approaches used by the recommender system Userbased approach, Item based approach, Hybrid recommendation approaches and related research in the recommender system. In the end we will show the main challenges and issues recommender systems come across.
\end{abstract}

Keywords: Recommendation system, Content based algorithm, Collaborative filtering algorithm

\section{Introduction}

Recommendation systems ( replacing "system" with a synonym such as platform or engine) are a subclass of information filtering system that seek to predict the 'rating' or 'preference' that a user would give to an item. The First recommender system was developed by Goldberg, Nichols, Oki \&Terry in 1992. Tapestry was an electronic messaging system that allowed users to either rate messages ("good" or "bad") Recommender system as defined by $\mathrm{M}$.

Deshpande and G. Karypis: A personalized information filltering technology used to either predict whether a particular user will like a particular item or to identify a set of $\mathrm{N}$ items that will be of interest to a certain user. Recommender systems work from a specific type of information filtering system technique that attempts to recommend information items (movies, TV program/show/episode, video on demand, music, books, news, images, web pages, scientific literature etc.) or social elements (e.g. people, events or groups) that are likely to be of interest to the user. Typically, a recommender system compares a user profile to some reference characteristics, and seeks to predict the 'rating' or 'preference' that a user would give to an item they had not yet considered. These characteristics may be from the information item-it may be analogous (the content-based approach) or the user's social environment (the collaborative filtering). The recommender system applies data mining techniques and prediction algorithms to predict useres interest on information, product and services user Recommender systems apply techniques and Retrieval. However, most of these systems can bear in their core an algorithm that can be understand as a particular instance of a data mining(DM) technique. The process of data mining consists of 3 steps, these are carried out in succession: Data Preprocessing, Data Analysis and Result Interpretation. Examples of recommender system are amazon.com, snapdeal.com, eBay [2], Levis [2], Moviefinder.com,

\section{Background}

Recommender systems typically produce a list of recommendations in one of two ways - through collaborative or content-based filtering. Content based algorithm recommender system [are the recommender system which work with profiles of users that are created at the beginning. A profile has information about a user and his/her taste. Taste is based on how user rated items.

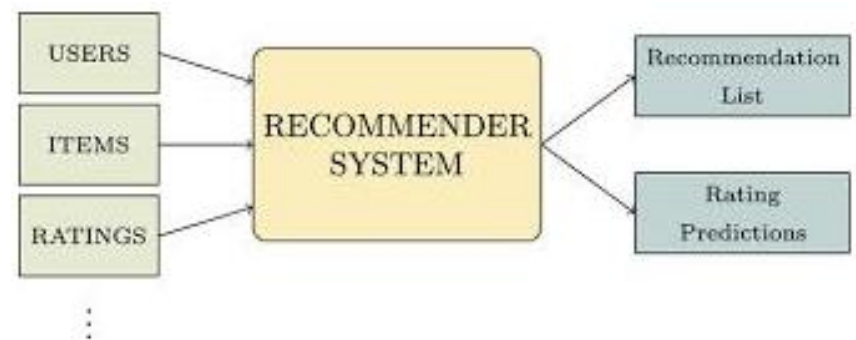

Figure 1: Recommendation System

In the recommendation process, the engine compares the items that were already positively rated by the user with the items he did not rate and looks for similarities. Those items that are mostly similar to the positively rated ones, will be recommended to the user. Collaborative filtering Algorithm[recommender system became one of the most researched techniques in the recommender systems since this approach was mentioned and described by Paul Resnick and Hal Varian in 1997. [1] The idea of collaborative filtering is in finding users in a community that share appreciations. If two users have same or almost same rated items in common, then they have similar tastes [11]. Such users build a group or a so called neighborhood. A user gets recommendations to those items that he/she has not rated before, but that were already positively rated by users in his/her neighborhood Various approaches of Collaborative Filtering are (1) Userbased approach,( 2) item based approach ,

User based approach: In the user-based approach, the users perform the main role. If certain majority of the customers has the same taste then they join into one group. Recommendations are given to user based on evaluation of items by other users form the same group, with whom he/she shares common preferences. If the item was positively rated by the community, it will be recommended to the user.

Item Based Approach: the taste of users remains constant or change very slightly similar items build neighborhoods based on appreciations of users. Afterwards the system generates recommendations with items in the neighborhood that a user would prefer. 


\section{International Journal of Science and Research (IJSR) \\ ISSN (Online): 2319-7064 \\ Index Copernicus Value (2013): 6.14 | Impact Factor (2015): 6.391}

\section{Literature Survey}

Borrow ideas of object typicality from cognitive psychology and propose a novel typicality-based collaborative filtering recommendation method named Tyco. A distinct feature of typicality-based CF is that it finds ,neighbors" of users based on user typicality degrees in user groups (instead of the corated items of users, or common users of items, as in traditional CF). To the best of our knowledge, there has been no prior work on investigating $\mathrm{CF}$ recommendation by combining object typicality. Tyco outperforms many CF recommendation methods on recommendation accuracy (in terms of MAE) with an improvement of at least $6.35 \%$ in Movie lens Data set. It can obtain more accurate predictions with less number of big-error predictions. . In Tyco, a user is represented by a user typicality vector which can indicate the user"s preference on each kind of items. A distinct feature of

Тусо is that it selects „neighbors" of users by measuring users" similarity based on their typicality degrees instead of co-rated items by users [8]

This model gave each user an option to adjust the diversity of their own recommendation list by using the prevalence rate and novelty rate parameters. Experiments using realworld rating datasets indicated the proposed model had effectively increased the recommendation diversity with little decrease in accuracy and surpassed the traditional collaborative filtering techniques .we find that user-based collaborative filtering technique is more inclined to recommend popular items and item-based collaborative filtering technique is more inclined to recommend long tail items The recommendation model we have proposed can accept users" feedback, so it meets the users" needs and provide more diverse recommendation result. Experiments using real-world rating datasets indicate that the proposed model effectively increases the recommendation diversity with little decrease in recommendation accuracy and surpasses the traditional collaborative filtering techniques. [9]

The performance of cluster ensembles for recommendation has not been fully examined. Thus, the aim of this paper is to assess the applicability of cluster ensembles to collaborative filtering recommendation. In particular, two well-known clustering techniques (self-organizing maps (SOM) and kmeans), and three ensemble methods (the cluster-based similarity partitioning algorithm (CSPA), hyper graph partitioning algorithm (HGPA), and majority voting) are used. The experimental results based on the Movie lens dataset show that cluster ensembles can pro-vide better recommendation performance than single clustering techniques in terms of recommendation accuracy and precision.[5]

Propose a collaborative filtering method to provide an enhanced recommendation quality derived from user-created tags. Collaborative tagging is employed as an approach in order to grasp and filter users" ${ }^{\text {ec }}$ preferences for items. In addition, they explore several advantages of collaborative tagging for data sparseness and a cold-start user. These applications are notable challenges in collaborative filtering. They present empirical experiments using a real dataset from del.icio.us. Experimental results show that the proposed algorithm offers significant advantages both in terms of improving the recommendation quality for sparse data and in dealing with cold-start users as compared to existing work. , collaborative tagging is becoming widely used as an important tool to classify dynamic content for searching and sharing [4]

Taking into account the interaction context of users will improve the relevancy of the recommendation process. However, only a few prior studies have tried to adopt context-awareness to the recommendation model. Although a number of studies have developed recommendation models using collaborative filtering (CF), few of them have tried to adopt both $\mathrm{CF}$ and other artificial intelligence techniques, such as genetic algorithm (GA), as a tool to improve recommendation results. In this study, we proposed a novel model (Context-Aware CF using GA) for location-based advertising recommendation. Our proposed model -CACFGA - is designed to perform CF by considering personal context information - location, time, and needs type. The CACF-GA model can be implemented to provide an improved mobile business model in the mobile advertising environment [6].

Introduce the social trust of the users into the recommender system and build the trust relation between them. The values of trust among users are adjusted by using the reinforcement learning algorithm. On the basis of this, a user trust-based collaborative filtering recommendation algorithm is proposed. It uses the combined similarity to generate recommendation, which considers not only the similarity between user profiles but user trust as well. Experimental results show that the proposed algorithm outperforms the traditional user-based and item-based collaborative filtering algorithm in recommendation accuracy.

User personality is introduced to improve the user model, and two personality-based collaborative filtering recommendations are proposed: one is to compute user similarity from the user personality perspective and select nearest neighbor, and then generates recommendation; another one is based on the personality-item rating matrix, and then make recommendation to the target users. These two ideas can well make up for the inadequacies of the current collaborative filtering recommendation system. In the meantime, the experiment result shows that user personalitybased collaborative filtering approach performs better than existing ones.[1]

\section{Related Research}

In practice, research paper recommender systems do not exist. However, concepts have been published and partly implemented that could be used for their something desired. Some authors suggest using collaborative filtering and ratings. Ratings could be directly obtained by considering citations as ratings or implicitly generated by monitoring readers" actions such as downloading a paper. Citation databases such as Cite Seer apply citation analysis (e.g. bibliographic coupling or co-citation analysis in order to identify papers that are similar to an input paper Scholarly search engines such as Google Scholar focus on classic text 


\section{International Journal of Science and Research (IJSR) \\ ISSN (Online): 2319-7064 \\ Index Copernicus Value (2013): 6.14 | Impact Factor (2015): 6.391}

mining and citation counts. Each concept does have disadvantages, which limits its suitability for generating recommendations. Recommender systems cannot identify related papers if different terms are used. Collaborative filtering in the domain of research paper recommendation is criticized for various reasons. Some authors claim that collaborative filtering would be ineffective in domains where more items than users exist. Others believe that users would be unwilling to spend time for explicitly rating research papers. Problematic with implicit ratings is that for obtaining the required data, continuous monitoring of the researcheres work is necessary, which raises privacy issues [20]. In general, collaborative filtering has to cope with the possibility of manipulation. Another drawback is that a critical mass of ratings and users is required to receive useful recommendations.

\section{Challenges and Issues}

\section{Cold start}

It ${ }^{\text {ee }}$ s difficult to give recommendations to new users as his/her profile is almost empty and he has not rated any items yet so his taste is unknown to the system. This is called the cold start problem. In some recommender systems this problem is solved with survey when creating a profile. Items can also have a cold-start when they are new in the system and haven "t been rated before. Both of these problems can be also solved with hybrid approaches. [1]

\section{Trust}

The voices of people with a short history may not be that relevant as the voices of those who have rich history in their profiles. The issue of trust arises towards evaluations of a certain customer. The problem could be solved by distribution of priorities to the users. [1]

\section{Scalability}

With the growth of numbers of users and items, the system requires more resources for processing information and forming recommendations. Most of resources is consumed with the purpose of determining users with similar tastes, and goods with similar descriptions. This problem is also solved by the combination of various types of filters and physical improvement of systems. Parts of numerous computations may also be implemented offline in order to accelerate issuance of recommendations online. [1]

\section{Sparsity}

In online shopping those have a huge amount of users and items there are almost always users that have rated just a few items. Using collaborative filtering and other approaches recommender systems generally create neighborhoods of users using their profiles. If a user has evaluated just few items then it's pretty difficult to determine his/her taste and he/she could be related to the wrong neighborhood. Sparsity is the problem of lack of information. [1]

\section{Privacy}

Privacy has been the most important problem. In order to receive the most accurate and correct recommendation, the system must acquire the most amount of information possible about the user, including demographic data, and data about the location of a particular user. Naturally, the question of reliability, security and confidentiality of the given information arises. Many online shops offer effective protection of privacy of the users by utilizing specialized algorithms and programs.[1]

\section{Conclusion and Future Work}

This paper presented the different techniques and algorithm to build the recommender system We also introduce different modern recommendation approaches like context-aware approaches, Semantic based approaches, cross-domain based approaches, peer to-peer approaches and cross-lingual approaches

.we also studied cold start problem[8].A recent research topic in the context of the recommender system is recommendations to group. We have also uncovered areas that are open to many further improvements, and where there is still much exciting and relevant research to be done in coming years.

\section{References}

[1] Zhicho Quan , "Collaborative Filtering Recommendation Based On User Personality", $6^{\text {th }}$ international conference on information management,2013.

[2] Reena Pagare, "Study Of Collaborative Filtering Recommendation Algorithm scalability Issue" ,international journal of computer application(09758887).2013

[3] Rong $\mathrm{Hu}$, Wanchun Dou, Jianxun Liu, "ClubCF:AClustering-Based Collaborative Filtering Approach for Big Data Application", IEEE Transactions onEmerging Topics in Computing, vol.2, no. 3, pp. 302-313, Sept. 2014,doi:10.1109/TETC.2014.2310485

[4] Heung-Nam Kim, Ae-Ttie Ji, Inay Ha, Geun-Sik Jo. "Collaborative filteringbased on collaborative tagging for enhancing the quality of recommendation", 15674223/\$ - see front matter _2009 Elsevier B.V. doi:10.1016/j.elerap.2009.08.004

[5] Chih-Fong Tsai Chihli Hung."Cluster ensembles in collaborative filterinz recommendation", apply soft computing 12(2012)1417-1425.

[6] Tuan Hung Dao, Seung Ryul Jeong, HyunchulAhn.” a novel recommendation Model of location-based advertising: context-aware collaborative filtering using GA Approch", expert system with application 39 (2012) 3731-3739.

[7] Hong yan ," Effective browsing technique based on behavioral collaborative filtering on social stream, $18^{\text {th }}$ international conference on knowledge based and intelligent information and engineering system,2014"

[8] Yi Cai ${ }^{-}$, Ho-fung Leung ${ }^{\dagger}$, Qing Li, Huaqing Min', Jie Tang and Juanzi Li ," Typically based collaborative filtering recommendation, knowledge and data engineering ,IEEE ,2014"

[9] Jing Wang, Jian Yin, "Combining User-based and Item-based Collaborative Filtering Techniques to Improve Recommendation Diversity",IEEE,2013

[10] http://infolab.stanford.edu

[11] file.grouplens.org 


\section{International Journal of Science and Research (IJSR) \\ ISSN (Online): 2319-7064}

Index Copernicus Value (2013): 6.14 | Impact Factor (2015): 6.391

[12] https://en.wikipedia.org

[13] Fuzhi Zhang, Long Bai ,Feng Gao ," A User TrustBased Collaborative Filtering Recommendation Algorithm”, SPRINGER ,2009

\section{Author Profile}

Mrs. Pinal Patel received the B.E degree in Computer Engineering from Ahmedabad Institute of Technology in 2014. She will complete her M.E in Computer Engineering from Silver Oak College of Engineering and Technology in 2016.

Ms. Pooja Jardosh received the B.E. degrees in Computer Engineering from silver oak collage of engineering and technology and received Master Degree in Information Technology from charotar university of science \& technology Institute under Gujarat Technological University. Currently she is working as assistant professor at Silver Oak College of Engineering and Technology. 